\title{
GPR surveying method as a tool for geodetic verification of GESUT database of utilities in the light of BSI PAS128
}

\author{
Marta Gabryś $^{1 *}$, Katarzyna Kryszyn ${ }^{1}$ and Łukasz Ortyl ${ }^{1}$ \\ ${ }^{1}$ Faculty of Mining Surveying and Environmental Engineering, AGH University of Science and Technology, \\ Mickiewicza Av. 30, 30-059, Cracow, Poland \\ *marta.gabrys@agh.edu.pl
}

\begin{abstract}
Intensified investment processes in construction have resulted in increased interest in the methods of efficient detection, verification and location of underground utility networks. In addition to the well-known pipe and cable locating equipment, which has increased its efficiency and reliability through the development of technologies, GPRs are becoming more and more popular.

This publication presents the results of the experimental research carried out with the use of GPRs manufactured by two different companies as well as the results of the verification of underground utilities in real conditions. The GPRs have worked in the mode of the real-time location of their own position using the GNSS system or robotic total stations. The GPR (Ground Penetrating Radar) surveys performed on a test field, consisting of 9 pipes with a known position, were aimed at assessing the accuracy of their identification on echograms. The utility line location errors were determined using three different combinations between the GPR and the locating instrument. It allowed the evaluation of the possibility of using these solutions for detection, verification and location of underground utility networks in the light of the Polish legal regulations and the British specification PAS 128.

The verification in real conditions was carried out in a typical urban space, characterised by an intense occurrence of underground utilities, that is, sewage systems, gas pipelines and power cables. It was based on the GESUT database captured from the county geodetic and cartographic documentation centre. The results of the visual analysis of the materials captured with the help of two measurement systems were described in detail, however, the verification was carried out only for one set of data. The authors have presented the procedure of processing echograms and detecting the location of pipeline axes based on their vectorisation. The authors of this research paper have performed a numerical analysis of the compliance of the profiles of utility lines with the information from the base map for two variants of the GPR data integration with the coordinates. The authors of this research paper have also presented an alternative concept of capturing the profile of a utility line in the field based on the processing of GPR data in 3D - the so-called C-scan. The conclusions summarise the possible factors affecting the surveying results and the methods of eliminating sources of errors, both for the GPR and geodetic data.
\end{abstract}

Key words: utility, ground penetrating radar, pipe detection accuracy, PAS128 


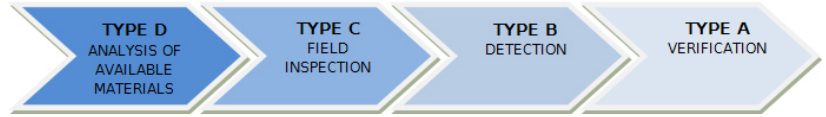

Figure 1. Levels of accuracy of locating underground utility networks according to BSI (2014)

\section{Introduction}

Ground penetrating radars have been widely used in geological, archaeological or forensic research and studies for many years. In recent years, it has also attracted the attention of designers and investors. Numerous problems related to the design and implementation of buildings or infrastructure result from the lack of reliable information on subsurface space, mainly utilities, that is, gas pipelines, waterworks, power or telecommunication cables (Jaw et al., 2018). Any work that involves earthworks entails a risk of damaging the pipelines, which threatens not only the finances of investors but, above all, the safety and comfort of citizens' lives. The legislative changes introduced in Poland have resulted in the requirement of digitising, that is, the GESUT database (Regulation, 2015; Act, $1989,2010)$, which is often implemented by the vectorisation of the available analogue materials, including the archival materials. With the information captured in this way, there is a common belief about its low reliability as to both horizontal and vertical location of utilities. The current standards (Regulation, 2011) describe the principles of direct surveys, however, there is no explicit reference to indirect methods of surveying hidden components of utility networks. The only record in the regulation concerns elevation issues and is extremely laconic (Par. 36 (1)(3)). The British specification PAS 128 (BSI, 2014) systematises the proper detection of utilities placed at a depth of up to 3 metres. It is based on a four-level division of the accuracy levels of the captured information - from D to A (Fig. 1). The higher the accepted type of identification, the greater the workload and the higher the costs of the works performed.

The basis for performing all the measurements is the survey type $D$, that is, the analysis of available documentation; the remaining levels are independent of each other. Survey type $C$ involves checking and, if necessary, supplementing the documentation with the components of the utility network identified during the field inspection. In survey type B, at least two detection techniques are used, including EML or GPR equipment. Georeference is determined in the national coordinate systems using GNSS receivers or total stations in real time, however, it is allowed to mark the profile of utilities on the ground and to perform surveys with classical techniques. The last one, type $\mathrm{A}$, assumes the most accurate inspection of the network by verifying the utilities in sewage sumps or inspection chambers, as well as by direct surveys performed in excavations. Each type of verification has its own quality level mark - QL, and the assumed accuracy of the acquired profile of the utility network (Tab. 1).

For the identification of the profile of utilities using one detection method, the QL-B3 quality level assumes obtaining the location accuracy not worse than $\pm 0.50 \mathrm{~m}$. According to the specification, after a suitably planned survey, data processing and detecting utilities with at least two methods, it is possible to achieve the QL-B1P accuracy level, that is, $\pm 0.15 \mathrm{~m}$ (horizontal) and $\pm 15 \%$ of the detected depth (vertical).

This publication presents an experiment carried out using the GPR method, which verifies if this method is able to achieve the accuracy of utility detection assumed by the British specification PAS 128 or required by the Polish standards, in particular, without a need to mark the profile of the network on the surface. Then, in the next part, the authors will demonstrate the result of verifying the profile of the underground utility network, carried out in real conditions in the Cracow city centre.

The performed surveys have created an opportunity to confront the systems of two GPR world leading brands. One of them - Mala RAMAC/CU II unit - was used for the experiment. The operation of another unit, fully dedicated to the performance of surveys aimed at locating underground utilities, has been described in more detail. The DS2000 GPR system is a new version of the proven solution, to which Leica has introduced significant improvements as far as geodetic surveys are concerned. The authors have verified, for example, the possibility of integrating echograms with coordinates in the local systems in real time thanks to the use of radio or Bluetooth transmission. The method of data processing, which refers to the classical way of capturing information on the profile of utilities based on echograms, has also been presented. In the spirit of software development, which significantly increases the capabilities of post-processing and GPR data analysis, the authors of this publication have presented the concept of capturing a vector profile of underground utility networks based on the $2 \mathrm{D}$ GPR data being processed into a 3D form, as well as capturing the horizontal slices of the area, the so-called C-scan image.

\section{Surveying equipment}

EM detectors are effective only for the detection of the utilities that conduct electric current, and therefore, generate a variable electromagnetic field. The performance of surveys is impeded in places with intensified occurrence of utilities, for example, in city centres or in the vicinity of industrialised areas. These problems have a huge influence on the time and quality of utility network verification, which made the contractors perform surveys using the GPR method. The work of the radar consists of emitting electromagnetic waves into the medium and recording the reflected wave at the boundary of the two media with varying electrical properties, for example, soil - PVC/steel pipe. Numerous design solutions for the GPR equipment are possible (Karczewski et al., 2011).

The operation of the RAMAC/CU II GRP system manufactured by Mala Geoscience was described in great detail in the doctoral dissertation of Ortyl (2006). In this dissertation, the robotic total station Leica TCRA 1102 was used for the direct location of GPR surveys in real time. In its basic version, the total station was able to define the position of the prism every $3 \mathrm{~s}$. The $0.67 \mathrm{~Hz}$ frequency of determining the position was achieved using additional total station software. The message in the NMEA protocol format (The National Marine Electronics Association) - the word LLQ (Leica Local Position and Quality) - containing the coordinates in the 2000 system, was transferred to the transmission port and then by radio to the software responsible for GPR data acquisition. Without the additional total station software, the reception of the positions using the NMEA protocol was possible only for the GNSS data. The RAMAC/CU II GPR saves geodetic coordinates with a frequency of $1 \mathrm{~Hz}$ and, depending on the measurement speed, up to 50 traces/second, which requires the interpolation of duplicated coordinates. Such a system of data acquisition and integration allowed to obtain the utility position error $m_{p}= \pm 0.13 \mathrm{~m}$ (Ortyl and Owerko, 2007).

With the advancement of technology, there are more and more instruments in the market with greater possibilities as far as the speed of data acquisition and its transmission is concerned. An example is the Leica Nova MS50 total station, whose specification states that it is possible to obtain a measurement frequency of approximately $10 \mathrm{~Hz}$ in a continuous measurement mode with the accuracy of the distance measurement of $3 \mathrm{~mm}+1.5 \mathrm{ppm}$. However, the tests carried out in real 
Table 1. Classification of quality levels (source: BSI (2014))

\begin{tabular}{|c|c|c|c|c|c|c|}
\hline \multirow{2}{*}{\multicolumn{2}{|c|}{ Type of study }} & \multirow{2}{*}{ QL } & \multirow{2}{*}{ Post-processing } & \multicolumn{2}{|c|}{ Location accuracy } & \multirow{2}{*}{ Additional data } \\
\hline & & & & Horizontal $^{1}$ & Vertical $^{2}$ & \\
\hline $\mathrm{D}$ & $\begin{array}{l}\text { Desktop utility records } \\
\text { search }\end{array}$ & QL-D & - & Undefined & Undefined & - \\
\hline $\mathrm{C}$ & Site reconnaissance & $\mathrm{QL}-\mathrm{C}$ & - & Undefined & Undefined & $\begin{array}{l}\text { Location of underground utilities in rela- } \\
\text { tion to above-ground objects. }\end{array}$ \\
\hline \multirow{4}{*}{ B } & \multirow{4}{*}{ Detection $^{3}$} & QL-B4 & No & Undefined & Undefined & $\begin{array}{l}\text { A presumably existing object - it has not } \\
\text { been detected despite the existing informa- } \\
\text { tion. }\end{array}$ \\
\hline & & $\begin{array}{l}\text { QL-B3 } \\
\text { QL-B3P }\end{array}$ & $\begin{array}{l}\text { No } \\
\text { Yes }\end{array}$ & $\pm 0.5 \mathrm{~m}$ & Undefined & $\begin{array}{l}\text { Horizontal position of the object detected } \\
\text { using one of the applied geophysical } \\
\text { techniques. }\end{array}$ \\
\hline & & $\begin{array}{l}\mathrm{QL}-\mathrm{B} 2 \\
\mathrm{QL}-\mathrm{B} 2 \mathrm{P}\end{array}$ & $\begin{array}{l}\text { No } \\
\text { Yes }\end{array}$ & $\begin{array}{c} \pm 0.25 \mathrm{~m} \text { or } \\
\pm 40 \% \text { of } \\
\text { detected depth }\end{array}$ & $\begin{array}{l} \pm 40 \% \text { of } \\
\text { detected depth }\end{array}$ & $\begin{array}{l}\text { Horizontal and vertical positions of the } \\
\text { object detected using one of the applied } \\
\text { geophysical techniques. }{ }^{4}\end{array}$ \\
\hline & & $\begin{array}{l}\text { QL-B1 } \\
\text { QL-B1P }\end{array}$ & $\begin{array}{l}\text { No } \\
\text { Yes }\end{array}$ & $\begin{array}{l} \pm 0.15 \mathrm{~m} \text { or } \\
\pm 15 \% \text { of } \\
\text { detected depth }\end{array}$ & $\begin{array}{l} \pm 15 \% \text { of } \\
\text { detected depth }\end{array}$ & $\begin{array}{l}\text { Horizontal and vertical positions of the } \\
\text { object detected by several applied } \\
\text { geophysical techniques. }{ }^{5}\end{array}$ \\
\hline A & Verification & $\mathrm{QL}-\mathrm{A}$ & - & $\pm 0.05 \mathrm{~m}$ & $\pm 0.025 \mathrm{~m}$ & $\begin{array}{l}\text { Horizontal and vertical positions of the up- } \\
\text { per and/or lower part of underground util- } \\
\text { ities. }\end{array}$ \\
\hline
\end{tabular}

\footnotetext{
${ }^{1}$ Horizontal location is to the centreline of the utility.

${ }^{2}$ Vertical location is to the top of utility.

3 For detection, it is a requirement that a minimum of GPR and EML techniques are used.

4 Electronic depth readings using EML equipment are not normally sufficient to achieve a QL-B2 or higher.

5 Some utilities can only be detected by one of the existing detection techniques. As a consequence, such utilities cannot be classified as a QL-B1.
}

conditions demonstrate the frequency of acquisition and transmission of coordinates closer to $5 \mathrm{~Hz}$ (Ortyl et al., 2015). This total station allows data to be sent to the communication port via the PseudoNMEA protocol.

Technological advancement has also greatly affected the design of GPRs, increasing the quality of the captured data as well as the speed of its collection. In the latest Leica DS2000 GPR system, it is possible to save nearly 400 traces/second, captured with two $700 \mathrm{MHz}$ and $250 \mathrm{MHz}$ antennas. The free reception of coordinates from total stations or GNSS receivers using radio transmission implemented in the GGA data format of the NMEA protocol has also become a standard.

\section{Research methodology}

\subsection{Test area I}

In order to verify the accuracy of locating utilities using the above-mentioned solutions, a study of three combinations of the systems were designed (Fig. 3). The studies were performed at the AGH test base at Mydlniki (Cracow), with 9 pipes of various diameters, made of different materials and embedded there at different depths (Ortyl, 2006). Their coordinates were captured by direct methods, constituting a proper reference for the results of indirect methods. The test base is located in a difficult terrain, overgrown with dense grasses in the summer, with a slope of approximately $4.5 \%$. The local control points were measured using the Leica GS16 receiver in the RTN surveying mode. Using the method of resection, the position of the total station was tied to these points with the accuracy of $\pm 0.01 \mathrm{~m}$. Based on the known profile of the pipes, two transverse profiles were designed, set out and marked, along which the antennas of the GPRs were directed (Fig. 2).

The first step involved the performance of a survey using the RAMAC/CU II GPR system with the antenna with the frequency of $500 \mathrm{MHz}$. The measurement of a single trace was triggered at the distance intervals of $0.03 \mathrm{~m}$. In order to improve the $\mathrm{S} / \mathrm{N}$ (signal to noise) ratio, the number of stacks equal to 32 and the sampling rate 10 times greater than the dominant frequency of the antenna was assumed. The time window with a length of $96 \mathrm{~ns}$ at a wave speed of $0.01 \mathrm{~m} / \mathrm{ns}$ allowed the penetration of the medium to a depth of approximately $5 \mathrm{~m}$. The Leica Nova MS50 total station and the 360 prism mounted on the dedicated adapter, $0.47 \mathrm{~m}$ above the ground, were used as a system for locating the position. The transmission of the coordinates in the 2000 system was carried out with Satteline 3ASd radio modems, using the PseudoNMEA protocol. Eight profiles were performed - two passes in opposite directions for both determined profiles.

Another combination included the compact DS2000 GPR system with two antennas with frequencies of $250 \mathrm{MHz}$ and $700 \mathrm{MHz}$. The same coordinate transfer system was adopted, as well as the locating instrument and the measurement step equal to $0.03 \mathrm{~m}$. The length of the time window was extended to $120 \mathrm{~ns}$. The available set did not have a dedicated adapter for the surveying instrument, therefore, the 360 prism on a twometre-high pole was attached to the handle of the trolley. One pass for both profiles was performed.

The last proposal for tying echograms to georeferencing was locating the coordinates for the DS2000 GPR system by the GNSS system in the RTN surveying mode. This method differed from the integration with the total station mainly with respect to the format and the way of data transmission. This was due to the fact that in this solution, thanks to the Bluetooth communication, the coordinates reached the GPR in the 


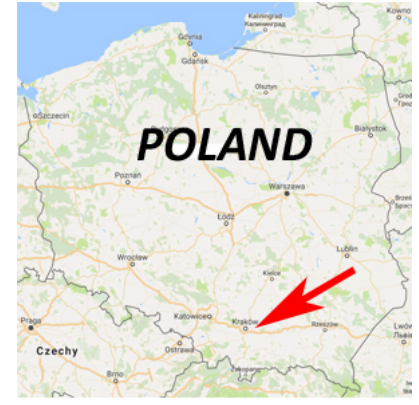

(a) Location of the research with reference to the area of Poland

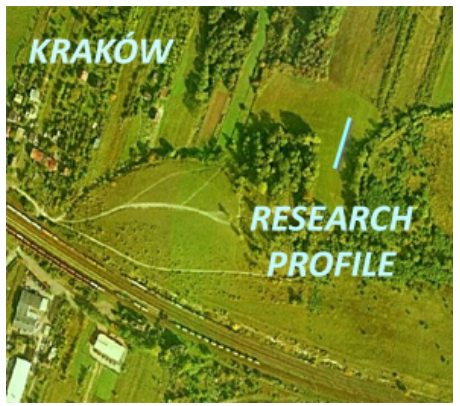

(c) Location of the research with reference to the city of Cracow

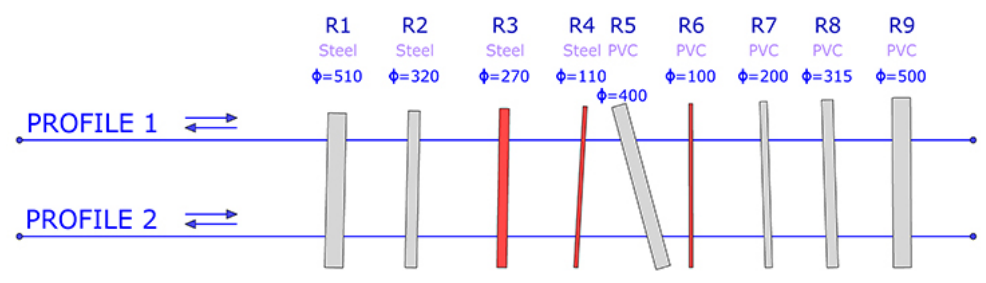

$-13.00 \mathrm{~m}-$

(b) The surveying profiles relative to the embedded pipes

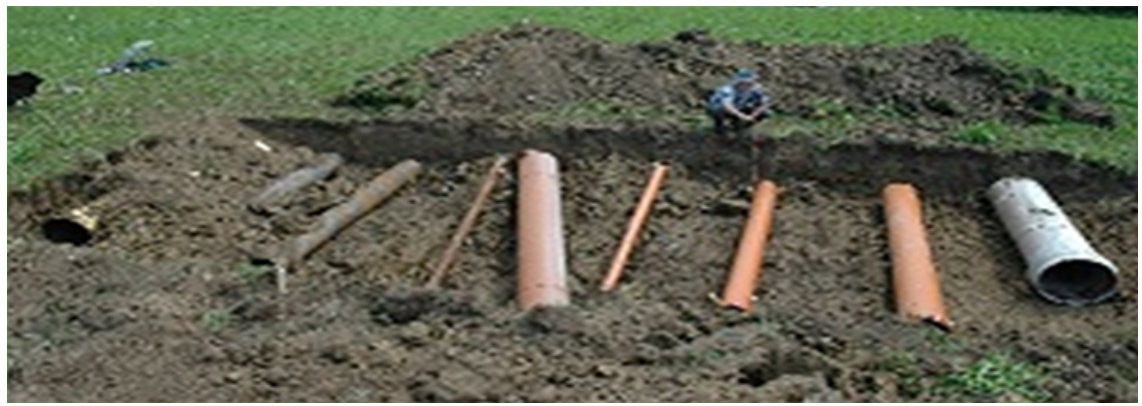

(d) The arrangement of the pipes before they were embedded

Figure 2. Draft of the location of the research and the arrangement of the pipes

WGS84 system converted to the UTM $34 \mathrm{~N}$ system.

In this study, the echograms from the three systems were subjected to a filtration sequence in the ReflexW programme. The signal was normalised, the background was removed and the bandpass frequency filters and the gain filter were used, which facilitated the identification and vectorisation of the pipes along the signals with the highest amplitude, forming a shape similar to a hyperbole. The results were exported to ASCII files where, according to the criterion of the shortest time of the beam pass, the numbers of the traces and the relative depths of the samples corresponding to the highest point of the pipe were determined.

In order to locate the measurement results from the Mala GPR system, the coordinates in the 2000 system were used, saved by the acquisition programme in the *.cor file. This allowed for simple import operations, the interpolation of the duplicated data and the integration of the echograms with the coordinates in the ReflexW programme (Fig. 4b). Although this programme has the ability to import data in the format appropriate for the IDS systems, the interpretation of an implicit georeferenced file caused the occurrence of the translation and scaling of the coordinates and, consequently, errors in the traces exceeding 10 metres. Therefore, the uNext programme was used to tie the coordinates with the results of the surveys of the DS2000 GPR system (Fig. 4a). For that data, the analysis of the distance of the pipeline determined by the GPR method relative to the sections representing the actual profile of the pipes was performed, as well as the analysis of the compliance of their embedment depths.

\subsection{Test area II}

Another survey was carried out in Cracow at Reymonta street, in the grassy area at the Faculty of Animal Breeding and Biology at the University of Agriculture. The choice of this specific area was dictated by its accessibility (without the need to stop road traffic) as well as the multiplicity and diversity of utilities, located parallel to each other, at distances not exceeding $3.5 \mathrm{~m}$. Based on the GESUT data obtained from the County Geodetic and Cartographic Documentation Centre, an area with 8 utility lines was selected (Fig. 5): sewage pipe (koD300), low pressure gas pipe (gnD300), medium pressure gas pipe (gs90), 4 medium voltage power cables $\left(\mathrm{eSA}_{1}, \mathrm{eSA}_{2}, \mathrm{eSA}_{3}, \mathrm{eSA}_{4}\right)$ and a bundle of 3 medium voltage power cables (3eSD). A grid of 26 profiles with a mesh of $1 \mathrm{~m} \times 1 \mathrm{~m}$ constituted the base for the surveys (Fig. 5). The longitudinal profiles of the grid were located parallel to the axis of the utility lines.

During the survey performed with the RAMAC/GPR CU II GPR system with a $500 \mathrm{~Hz}$ antenna, as in the case of the experimental research, the positioning was conducted with the MS50 total station, because the data of this solution was analysed for compliance with the available documentation. The DS2000 GPR was also used for the surveys, but without the possibility of real-time data positioning, so they were used only for the visual analysis of the echogram quality.

In order to determine the compatibility of the GPR data with the existing cartographic study, first of all, it was necessary to capture information on the profile of the utilities based on the acquired echograms. In order to facilitate the initial process of utility network identification, the GESUT documentation was used, based on which the approximate distances of the subsequent utilities were determined from the origins of the measurement profiles. The precise positions of the points representing the axes of the pipes on the echograms were captured based on the vectorisation of the signal with the highest amplitude and the identification of their tops according to the shortest time criterion. Finally, the profile of the straight line segments of the networks was determined using linear regression with the minimised observation distance from the straight line (Fig. 6). The adjustment was made for the two variants of determining the coordinates of the pipe tops:

i. based on the total station data,

ii. based on the interpolation of the coordinates along the profiles based on the knowledge of the origins and end posi- 


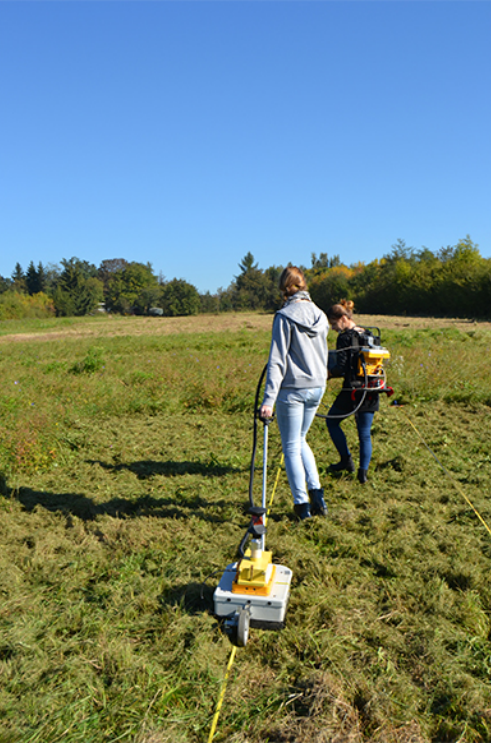

(a) Mala RAMAC/CU II GPR with a 360 prism

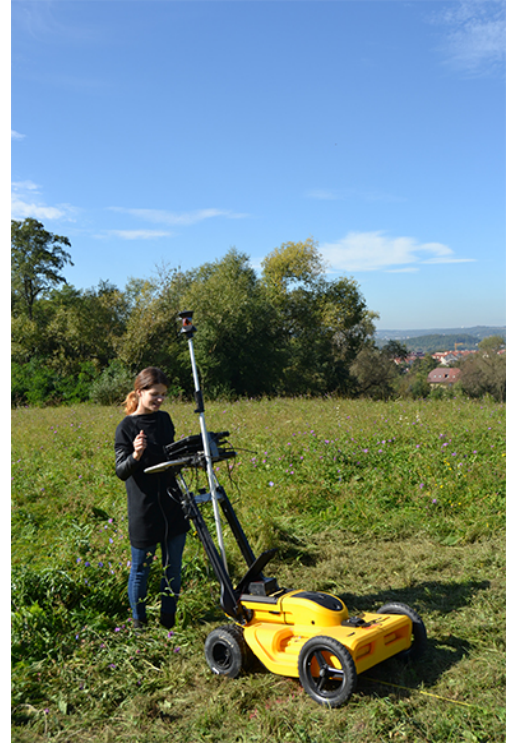

(b) DS2000 GPR localised by MS 50 total station (c) Leica GS16 GNSS receiver

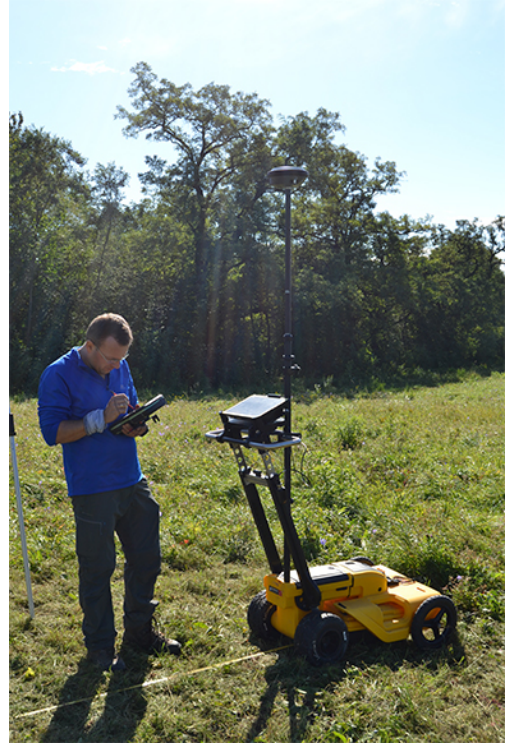

Figure 3. Surveying systems used

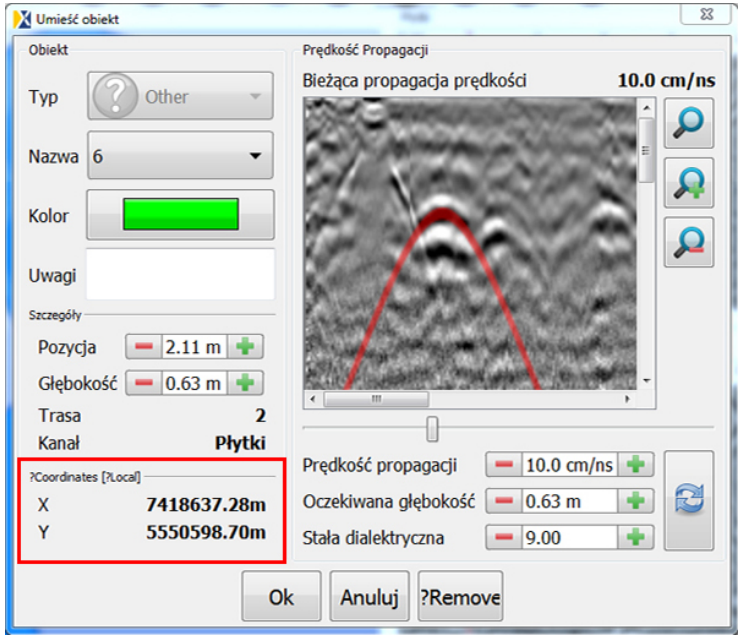

(a) Effects of integration of coordinates with echograms in uNext

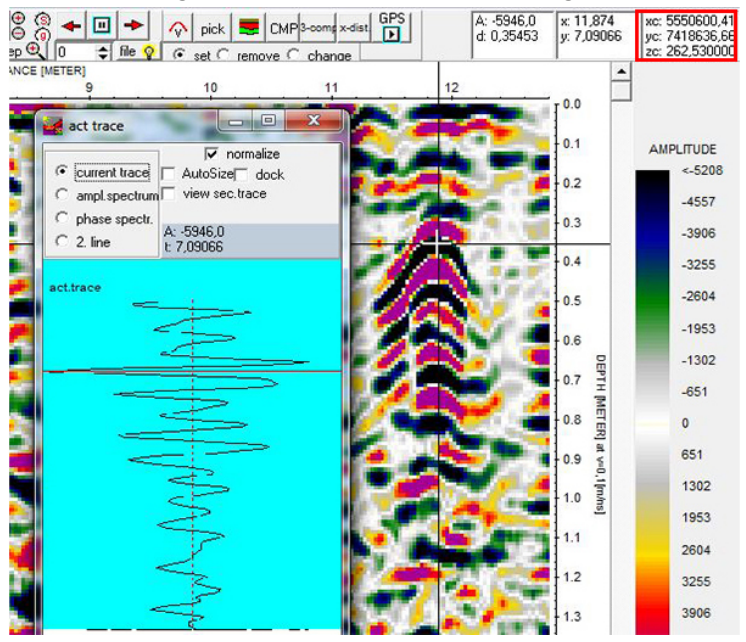

(b) Effects of integration of coordinates with echograms in ReflexW

Figure 4. Effects of integration of coordinates with echograms

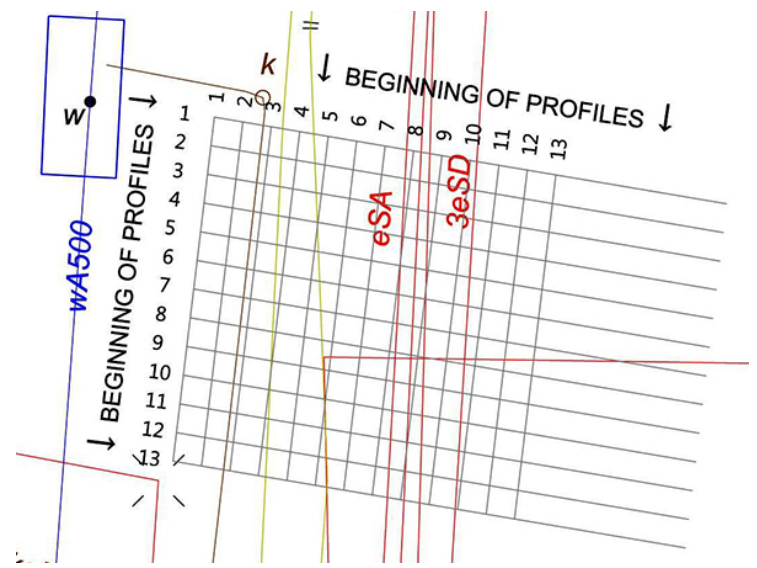

Figure 5. Arrangement of measurement profiles on available cartographic materials

tions and the assumed measurement step.

The distances between the approximate profiles of the utilities and the available documentation have formed the basis for the planar verification of the network compliance. The in-depth profile of the utilities along the test sections was also analysed.

The last stage of the study involved a visual comparison of the quality of the data captured with the use of the $500 \mathrm{MHz}$ Mala antenna, as well as the $250 \mathrm{MHz}$ and $700 \mathrm{MHz}$ Leica antennas, for the effectiveness of utility detection.

\section{Results of the study}

\subsection{Test area I}

Before presenting the numerical data illustrating the accuracy of the systems during the performance of the experiment in Mydlniki, it should be noted that the echograms of the DS2000 GPR system are characterised by a better quality and contrast of images, which has allowed to identify the pipes with $50 \%$ efficiency for both profiles. In the case of the Mala system, out of the expected 36 observations for a single profile, 15 and 


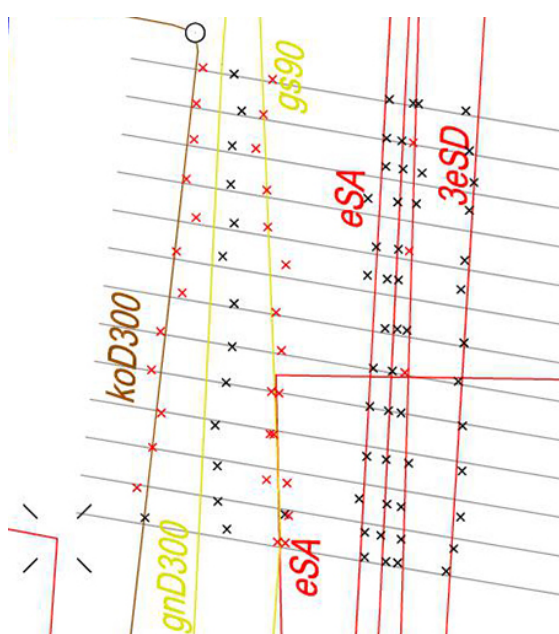

(a) Marking the tops of the pipes identified on (b) echograms on the map

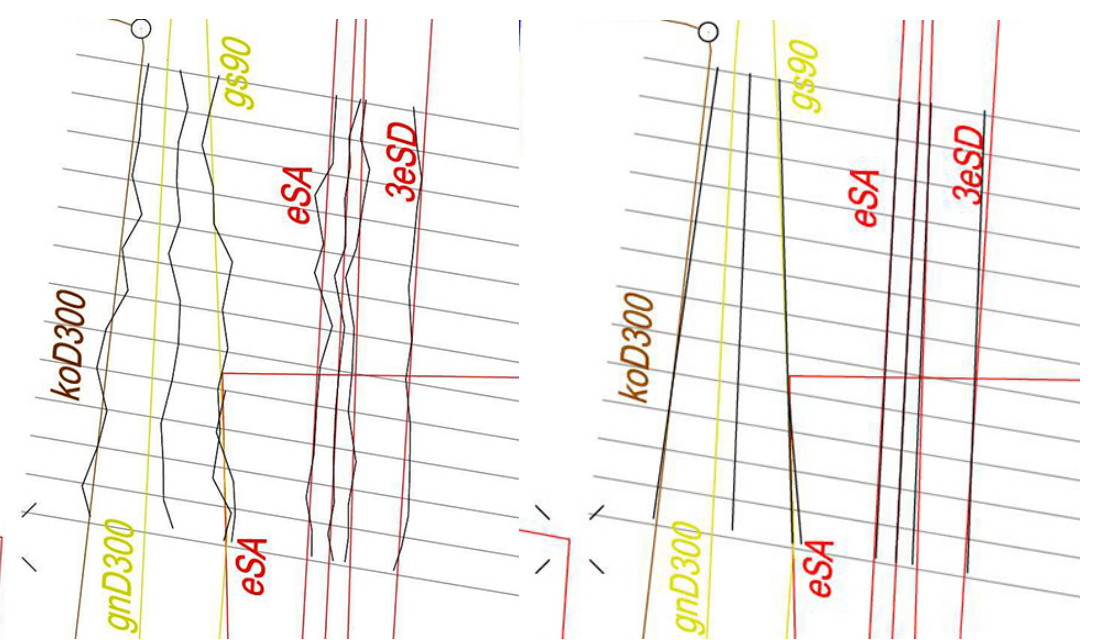

line
Determining a regression line for each utility line

Figure 6. Scheme of detecting the profile of utilities based on the GPR method

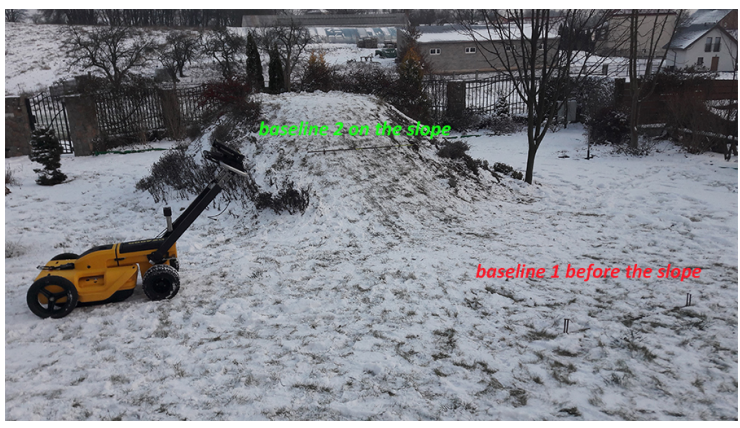

Figure 7. Location of the baselines of the supplementary measurement relative to the hill with a $45 \%$ slope

7 pipe tops were reliably identified for profile 1 and profile 2 , respectively. None of the echograms, regardless of the system used, identified the pipes R3, R4 or R6.

The mean square position error of the pipe tops identified on the RAMAC/CU II GPR echograms relative to their base profile was $\pm 0.10 \mathrm{~m}$. This error largely results from the necessity of interpolating the coordinates in places where there was a delay in radio data transmission or because the acquisition programme duplicated the captured coordinates for several subsequent traces. For the DS2000/TS and DS2000/GNSS combinations, this error assumed the values of $\pm 0.23 \mathrm{~m}$ and $\pm 0.24 \mathrm{~m}$, respectively. The errors induced by the Leica system significantly exceed the error obtained by the Mala system results. Thus, the errors of determining the position of this GPR in the direction transverse to the measurement profiles were also determined, and they reached $\pm 0.29 \mathrm{~m}$ and $\pm 0.19 \mathrm{~m}$. Such distribution of errors, both relative to the pipes and to the profiles, may point to the influence of a slope on determining the position of a high-placed target which, with the height of approximately $2.20 \mathrm{~m}$ above the ground and a slope of $4.5 \%$, induces an error of $\pm 0.01 \mathrm{~m}$.

In order to ensure that such conclusions were right, another test was carried out to supplement the experiment, in which the coordinates of the baselines located before and on the slope of the hill with the slope of $45 \%$ were determined in the RTKRTN measurement mode (Fig. 7)

When approaching the baselines with the GPR, the coordinates were read out in the uNext programme, however, this time the data was also processed using the available dedicated software. GRED HD is a powerful GPR data processing tool created by the IDS GeoRadar brand. The programme enables the creation of advanced 3D data analysis and visualisation as well as conversion of vectorisation to CAD formats. Leica has implemented similar solutions in its own DX Office Vision software, dedicated to the detection and digitalisation of utility lines based on the DS2000 GPR surveys.

The coordinates captured directly from the acquisition software were located at a distance of over $1.08 \mathrm{~m}$ from the baseline 2, which proves that no pole tilt adjustment was included in the programme. The processing of the data in the GRED HD programme improved the results, but with such a large slope of the terrain, differences of $0.36 \mathrm{~m}$ were obtained from the actual position of the baseline. The DX Office Vision programme proved to be the best tool in terms of the accuracy of integration of surveying data and echograms. The baseline coordinates obtained in it were located at a distance of $0.15 \mathrm{~m}$ from the actual profile of the line.

The parameter of the in-depth location of utilities is as important as their horizontal position for designing investments associated with the area where these utilities are located. During the analyses, there was a high repeatability of the results for each used systems. For the Mala system, there was a $78 \%$ total consistency of the depth of the pipe tops read out from the echograms for at least 2, and in some cases even 4 different passes; for the others, the differences did not exceed the value of $0.01 \mathrm{~m}$. For DS2000, the discrepancies between the two passes fell within $0.01 \mathrm{~m}-0.03 \mathrm{~m}$. Despite such high internal accuracy of both systems, the differences between them reached even $0.06 \mathrm{~m}$ and the deeper the pipe was embedded, the greater the difference noted. It is worth emphasising, however, that for the Mala system, having taken into account the terrain elevation, the differences in the value of the $\mathrm{Z}$-coordinate of the pipe tops did not exceed $0.02 \mathrm{~m}$.

The knowledge of the exact material characteristics of the pipes embedded in the test field allowed us to confirm the dependence of the polarisation of the wave reflected from the material of the pipes. The Mala system recorded positive values of amplitudes of the signal reflected from the steel pipes, and negative values for the PVC pipes (Radzevicius and Daniels, 2000). The reverse polarity was demonstrated by the signal emitted by the antennas of the DS2000 system (Fig. 8). 


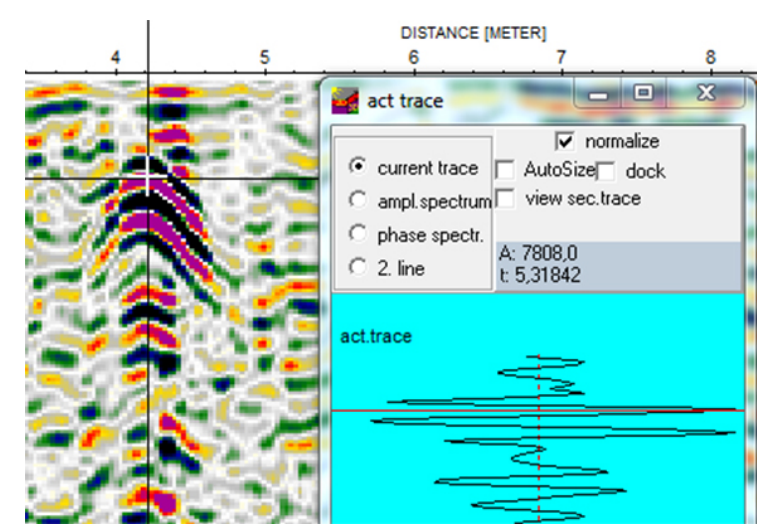

(a) measured with Mala RAMAC/CU II GPR

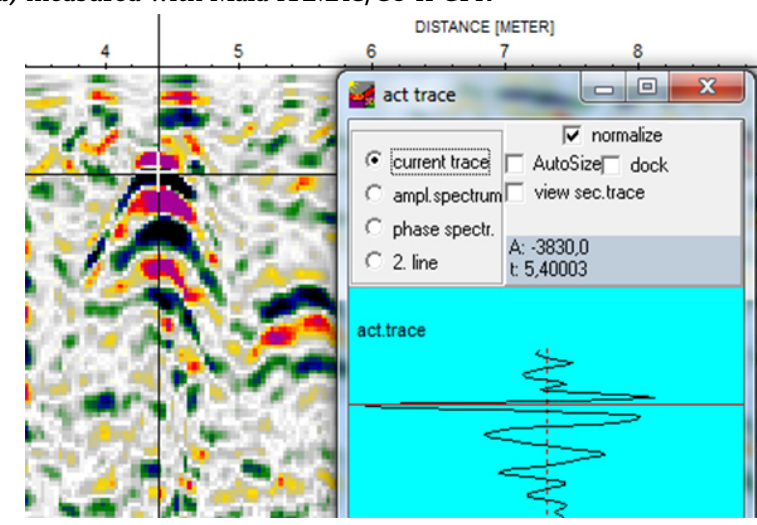

(b) measured with DS 2000

Figure 8. Polarisation of the wave reflected from the R1 pipe

\subsection{Test area II}

Verification of the results of the GPR surveys in relation to the GESUT cartographic data should have been started with a visual evaluation of the echograms. This is a preliminary, but very important part of the analysis, because the high amplitude signals are identified and classified at this stage. The data captured at Reymonta street turned out to be extremely difficult to interpret. Under favourable conditions of a geological substrate, the signal reflected from the upper surface of the linear utility should have the shape of a hyperbole. In the case of RAMAC/GPR CU II echograms, on the traces transverse to the utility lines, uniformly interpretable signals come only from the bundle of the power lines (3eSD), the gas pipe (gnD300) and the unidentified utility line located below the $3 \mathrm{eSD}$ bundle. For the power cables $\left(\mathrm{eSA}_{2}, \mathrm{eSA}_{3}, \mathrm{eSA}_{4}\right)$ there was a strong, elongated signal resulting from mutual interference of the reflected signals, so it was impossible for individual components to be visually distinguished. The power line $\left(\mathrm{eSA}_{1}\right)$ with the profile coincident with the gas pipeline (gs90) within the profiles 9-13 (Fig. 9) was not identified.

It could therefore be supposed that this power cable does not really exist. The analysis of the longitudinal profiles (Fig. 10) revealed the occurrence of a fragment of this utility line after changing its profile from the meridian to the latitudinal one, so this hypothesis was rejected in favour of another one that the gas pipe probably covers this power cable.

For the other utility lines, that is, koD300 and gs9o, there was no signal unambiguous for interpretation, so the fragments of the echograms were distinguished based on the documentation. The in-depth analysis of the signals with a strong response in the places where the sewage pipe was likely to occur, has demonstrated rapid changes in the depth of its em-
Table 2. Errors of fitting MNK regression lines [m]

\begin{tabular}{lrrrrrrr}
\hline \multicolumn{1}{c}{ Method } & gnD300 & gs90 & $\mathrm{eSA}_{1}$ & $\mathrm{eSA}_{2}$ & $\mathrm{eSA}_{3}$ & $\mathrm{eSA}_{4}$ & $3 \mathrm{eSD}$ \\
\hline GPR-MS50 & 0.15 & 0.21 & 0.17 & 0.17 & 0.11 & 0.13 & 0.12 \\
Distance & 0.14 & 0.17 & 0.14 & 0.15 & 0.15 & 0.15 & 0.09 \\
\hline
\end{tabular}

Table 3. Distances of regression lines relative to cartographic materials $[\mathrm{m}]$

\begin{tabular}{lrrrrrrr}
\hline $\begin{array}{l}\text { GPR-MS50 } \\
\text { method }\end{array}$ & gnD300 & gs90 & $\mathrm{eSA}_{1}$ & $\mathrm{eSA}_{2}$ & $\mathrm{eSA}_{3}$ & $\mathrm{eSA}_{4}$ & $3 \mathrm{eSD}$ \\
\hline Min & 0.41 & -0.04 & -0.04 & -0.01 & -0.01 & -0.13 & -0.22 \\
Max & 0.53 & 0.09 & 0.24 & 0.05 & 0.03 & 0.03 & 0.08 \\
Mean & 0.47 & 0.02 & 0.10 & 0.02 & 0.01 & -0.05 & -0.06 \\
\hline $\begin{array}{l}\text { Distance } \\
\text { method }^{1}\end{array}$ & $\mathrm{gnD} 300$ & $\mathrm{gs} 90$ & $\mathrm{eSA}_{1}$ & $\mathrm{eSA}_{2}$ & $\mathrm{eSA}_{3}$ & $\mathrm{eSA}$ & $3 \mathrm{eSD}$ \\
\hline Min & & & & & & & \\
Max & 0.37 & -0.14 & -0.11 & -0.23 & -0.21 & -0.25 & -0.39 \\
Mean & 0.46 & 0.09 & 0.16 & -0.11 & -0.11 & -0.16 & -0.10 \\
\hline & 0.42 & -0.04 & 0.02 & -0.17 & -0.16 & -0.20 & -0.24 \\
\hline
\end{tabular}

${ }^{1}$ Distance: profile from documentation - approximated profile $[\mathrm{m}]$

bedding, reaching $0.60 \mathrm{~m}$ on a one-metre-long section. In the execution of pipelines, especially general sewage systems, no slopes of $60 \%$ were applied. Therefore, this pipeline profile was considered incorrect, and the koD300 sewage pipe impossible to be detected only based on the available data.

The coordinates of the individual traces are encumbered with the error of their positioning method, which results in obtaining a broken axis of the utility line based on the vectorisation of the echograms, which forces the approximation of the shape of the axis to a more real straight line. Table 2 contains the values of the regression line fitting errors for the coordinates determined by the MS50 total station and for the coordinates interpolated along the profiles. Table 3 illustrates the values of the distances of the determined profiles relative to the base map. The method of positioning data by interpolation of the coordinates is less accurate (reveals the influence of the calibration of the encoder measuring the distance from the baseline of the profile origin), which is illustrated not only by the tabular data but also the visual consistency of the profiles. The limit values obtained for both methods do not exceed $0.30 \mathrm{~m}$ in most cases. The exception is the gas pipe gnD300, for which the offset of the measurement data from the base data is about $0.50 \mathrm{~m}$. This discrepancy is of a constant nature over the entire length of the measurement; so, it can be inferred that the position of this pipeline in the GESUT database is incorrect.

The GPR survey carried out with the use of the Mala system has confirmed the location of the utility lines: $\mathrm{eSA}_{2}, \mathrm{eSA}_{3}, \mathrm{eSA}_{4}$, $3 \mathrm{eSD}$ and gs90; it has indicated the probable correct profile of the gnD300 pipeline; it has detected an unidentified power cable located below $3 \mathrm{eSD}$; it has confirmed the existence of $\mathrm{eSA}_{1}$; and it has not confirmed koD300.

Contrary to the expectations (Reichel, 2017), the data captured by the DS2000 GPR at Reymonta street were characterised by slightly lower quality. On the echograms of the $700 \mathrm{MHz}$ antenna, there were no signals of reflection from the pipelines koD300, gs90, gnD300 or the alleged utility line identified, thanks to the RAMAC/CU II GPR. In the part of the echogram that is near the surface, the resolution and contrast were satisfactory, however, below the depth of $1 \mathrm{~m}$, the data were strongly noisy and the useful signals could not be distinguished. The echograms of the $200 \mathrm{MHz}$ antenna were a good complement to the data at lower depths (Fig. 12). 


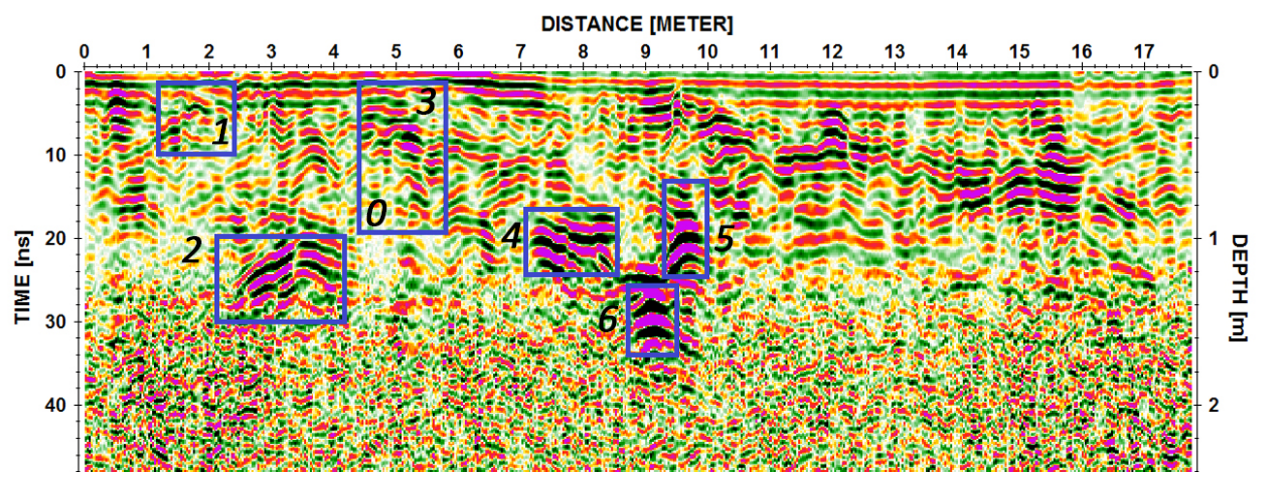

Figure 9. Classified echogram of the transverse trace - profile 12. Numbers of the areas marked on the echograms correspond to: 0 - eSA1; 1 - koD300; 2 - gnD300; 3 - gs90; 4 - eSA2, eSA3, eSA4; 5 - 3eSD; 6 - unidentified on cartographic materials utility line.

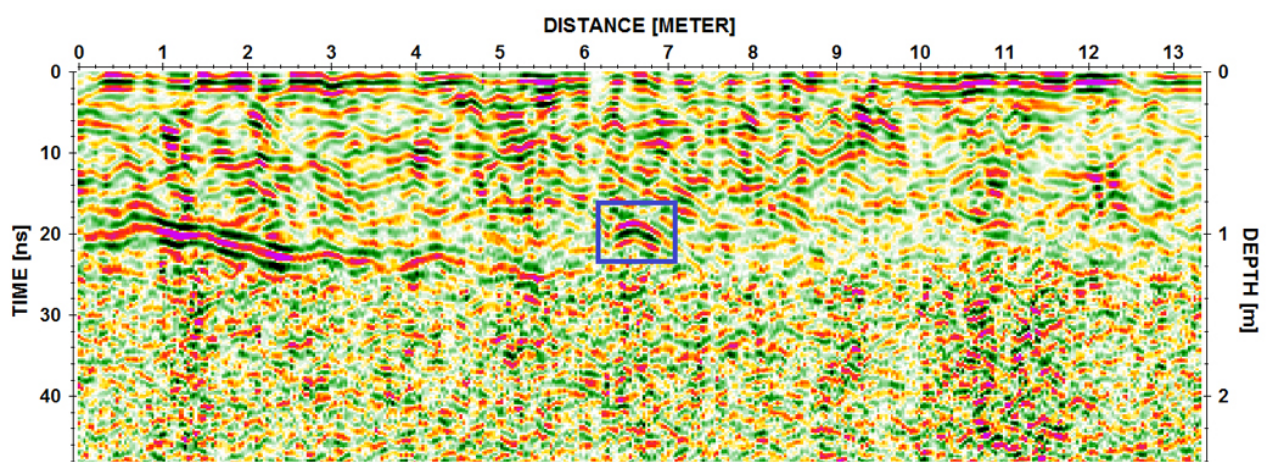

Figure 10. Echogram of the longitudinal trace - profile 10. The marked area of the occurrence of the eSA1 power line.

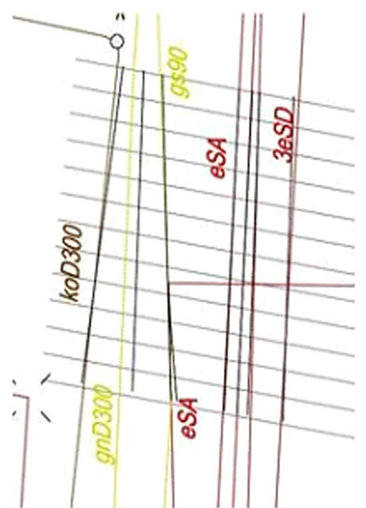

(a) GPR-MS50 method

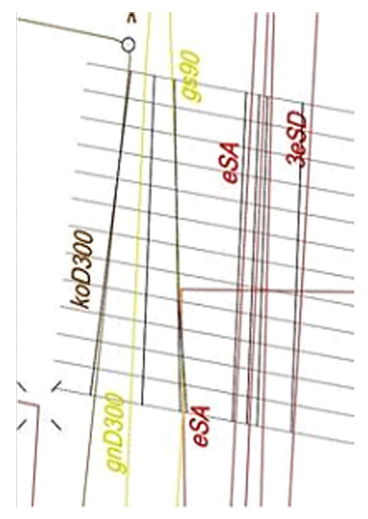

(b) Distance method

Figure 11. Obtained regression lines

\subsection{Vectorisation of the profile of underground utility networks on the GPR horizontal slice (C-scan)}

Capturing the profile of the utility network based on the vectorisation of echograms on rectilinear segments has yielded very good accuracy results. However, this is a multi-stage and time-consuming process. The longer the length of the utility line, the greater the effort required to perform repetitive activities, that is, analysis and vectorisation of subsequent echograms - B-scan. Only after the data has been processed for the entire segment, is it possible to perform the approximation of the axis profile. According to the authors of this publication, for the needs of the GESUT database, this process can be replaced by the vectorisation of the profile of the utilities identifiable on the horizontal slices of the C-scan of the 3D model performed at the appropriate depths (Fig. 13).

The quality of $2 \mathrm{D}$ data processing into a three-dimensional

model depends largely on the selected interpolation parameters (Lu et al., 2018) and the adopted coordinate system. There are two main ways of positioning echograms in space. The processes and problems described here are adequate for the ReflexW software, in which the processing has been carried out.

The first method involves the creation of a $3 \mathrm{D}$ model in a local system, for which the point of origin and the $\mathrm{X}$ axis are determined by the first profile of the model. The origins of subsequent echograms are arranged in accordance with the assumed profiling step along the $\mathrm{Y}$ axis. The interpolation is performed along the axis of the adopted system. This method is most effective in the event of the maximum fulfilment of conditions such as: the parallelism and equidistant increment of the subsequent profiles, the measurement step and the number of traces in the profile. The interpolated data fills tightly and closes within the boundaries of the studied area (Fig. 13).

The second method is to use the georeference for positioning echograms. The interpolation is performed along the main axes of the global system in which the GPR data are embedded. The selection of the interpolation parameters was carried out based on the tests of subsequent variants, since the acceptance of the values recommended by the manufacturer, based on the relative distribution of the profiles and the measurement step, would result in capturing data with significant deficiencies in the model continuity (Fig. 14). In addition, the programme was extrapolated on the edges of the area.

The model interpolated based on echograms and their coordinates can be arbitrarily cut along its main axes, also generating horizontal sections with a given depth and thickness. Strong signal reflections can be emphasised by further filtrating transformations and they can be exported to the geoTIFF form. It should be remembered that the ReflexW programme operates in a mathematical system, and therefore, it is necessary to calibrate it (regardless of the positioning method chosen) in the programmes that operate on surveying systems. 


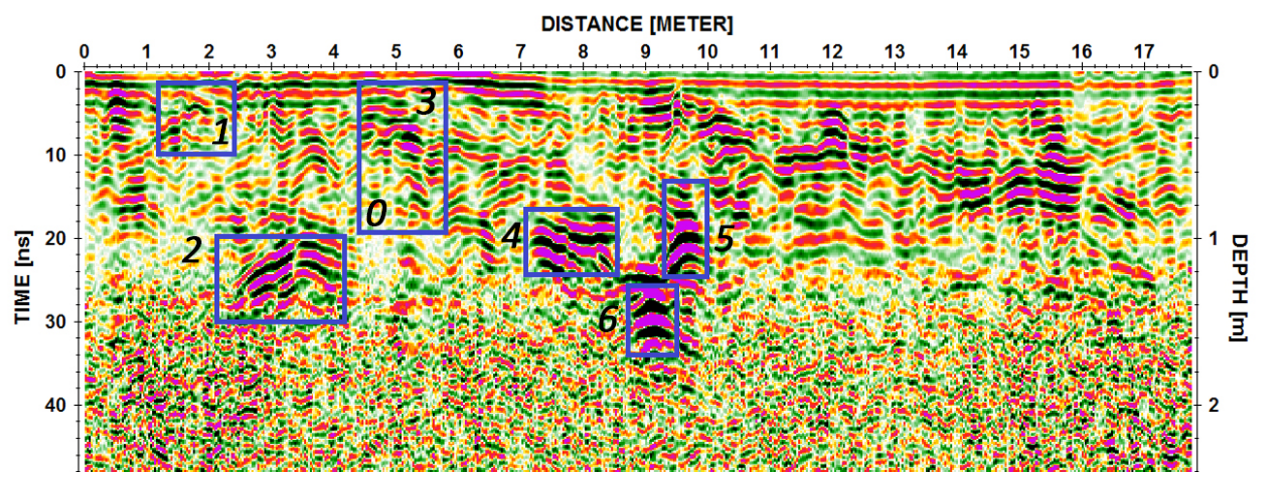

(a) Profile acquired with $500 \mathrm{MHz}$ antenna of the Mala system

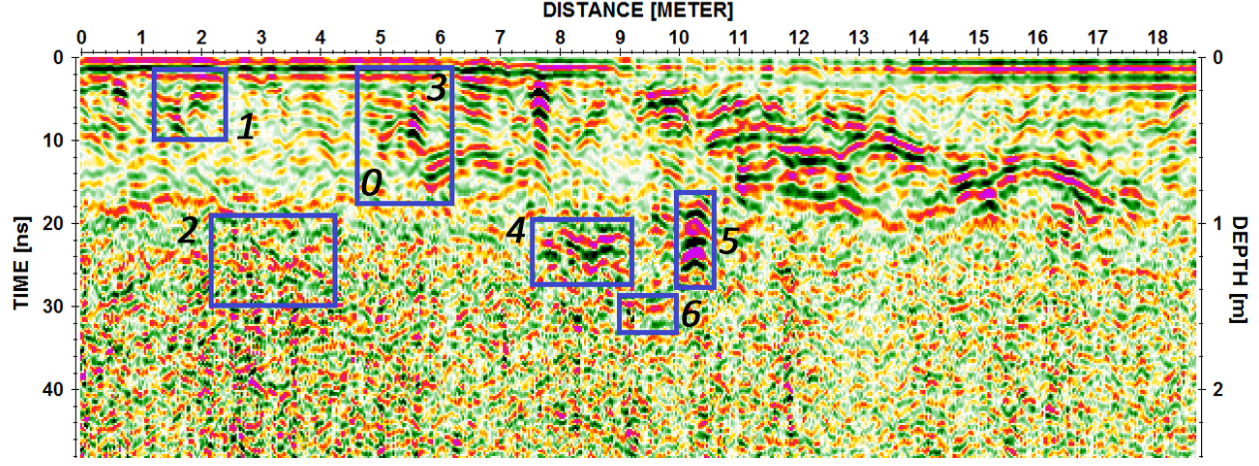

(b) Profile acquired with $700 \mathrm{MHz}$ antenna of the DS200 system

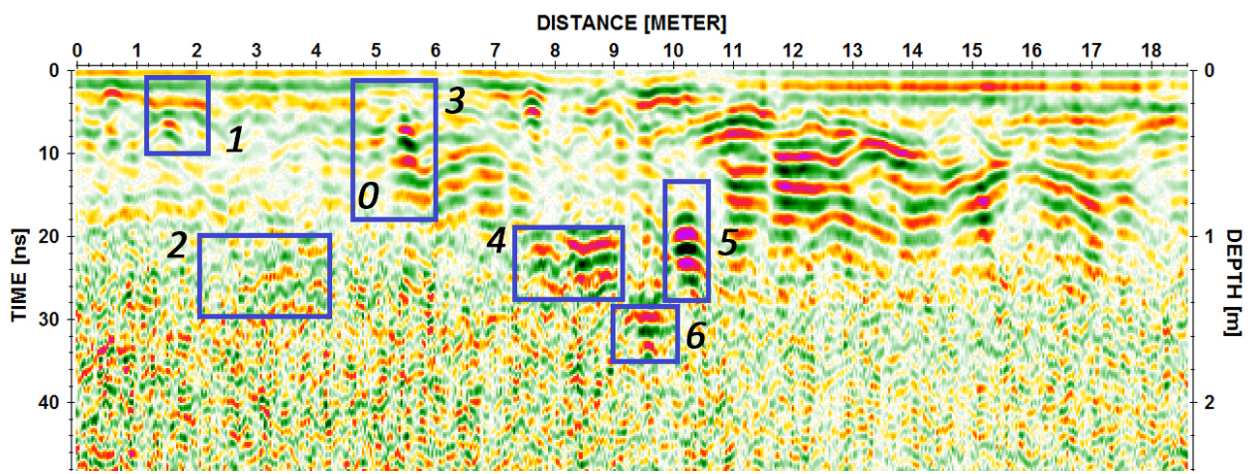

(c) Profile acquired with $250 \mathrm{MHz}$ antenna of the DS200 system

Figure 12. Echograms for the profile 12

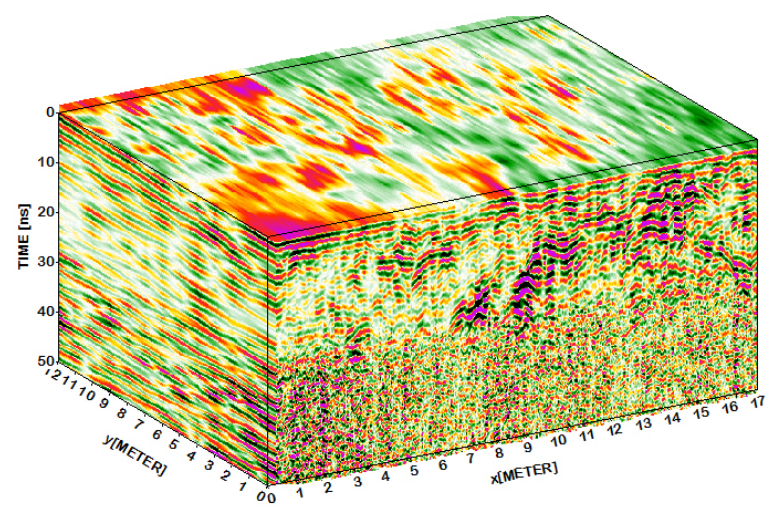

Figure 13. 3D model created based on 2D echograms (B-scan)
Most CAD programmes have the ability to load *.TIFF files and use them as references to create cartographic studies regarding, for example, vectorisation of the axes of utility lines (Fig. 15). The differences obtained between the axes and the regression lines, based on the coordinates of the subsequent tops of pipes in the studied area do not exceed $\pm 0.30 \mathrm{~m}$.

\section{Summary and conclusions}

In the light of the BSI PAS128 specification, the detection of utility lines using the GPR method meets the requirements of the highest accuracy group QL-B1P. An appropriate configuration of the GPR equipment and surveying instruments is able to ensure the horizontal accuracy of determining the coordinates of utility lines below $\pm 0.15 \mathrm{~m}$. The discrepancies in the determination of the heights of the lines between the two systems did not exceed $10 \%$ of the depth of the pipe embedment. Some sources of GPR survey location errors, that is, the instability of the measuring pole or the influence of a slope, can be eliminated by using dedicated hardware design solutions and using software that takes into account appropriate geometric 


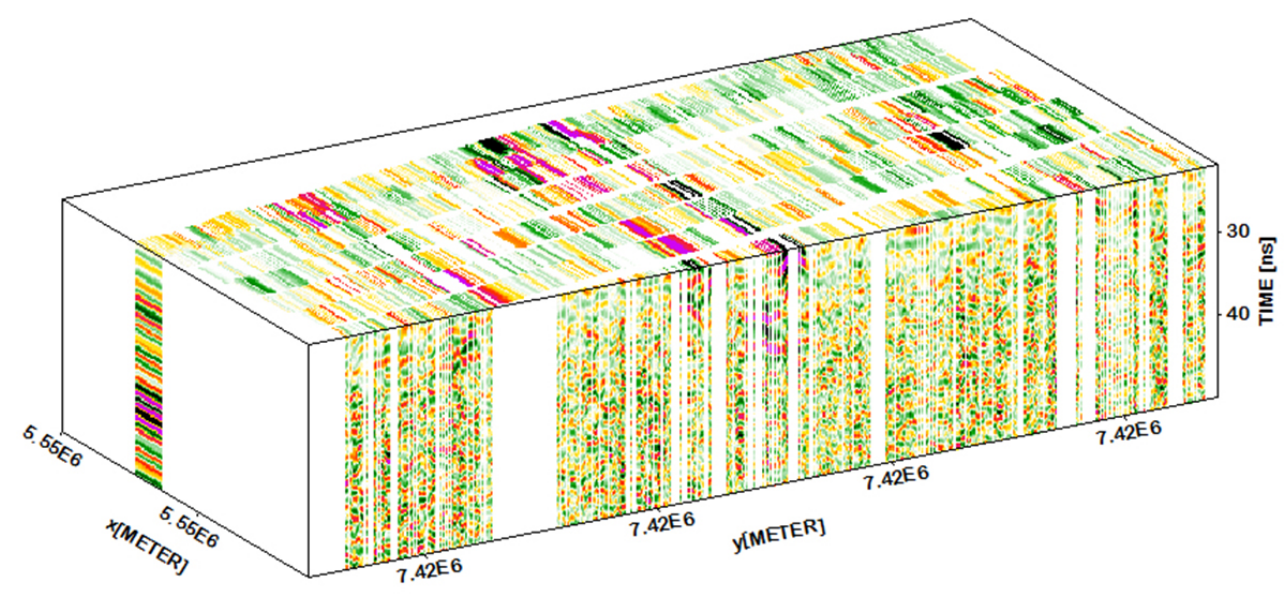

(a) Model created based on parameters recommended by the manufacturer

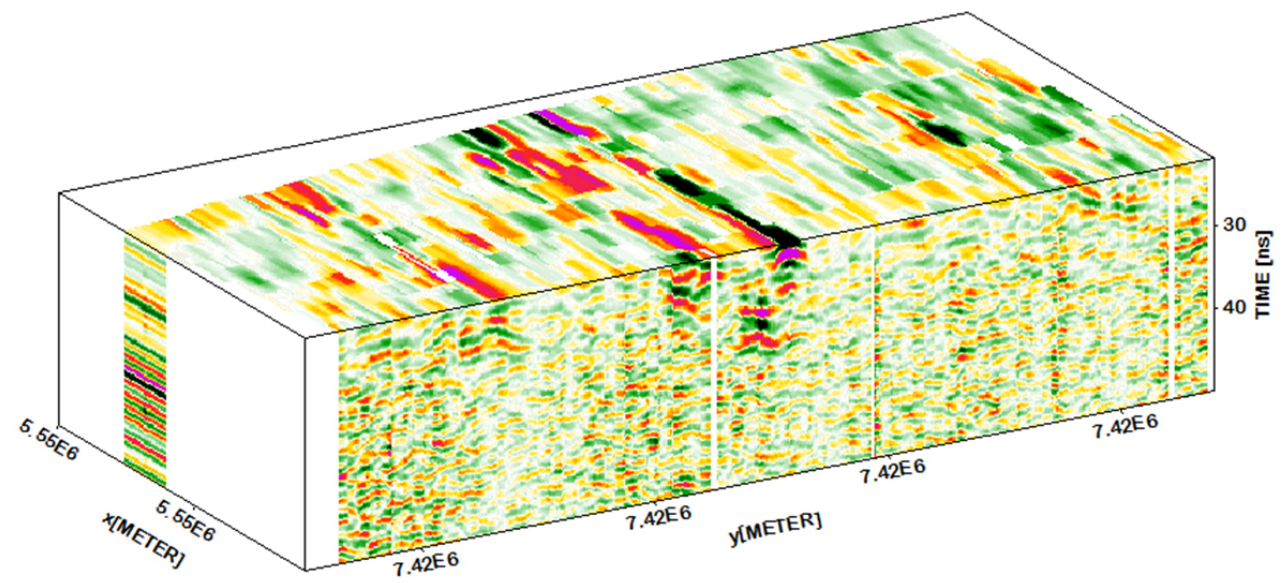

(b) Model created based on parameters selected empirically

Figure 14. 3D model with regard to georeference

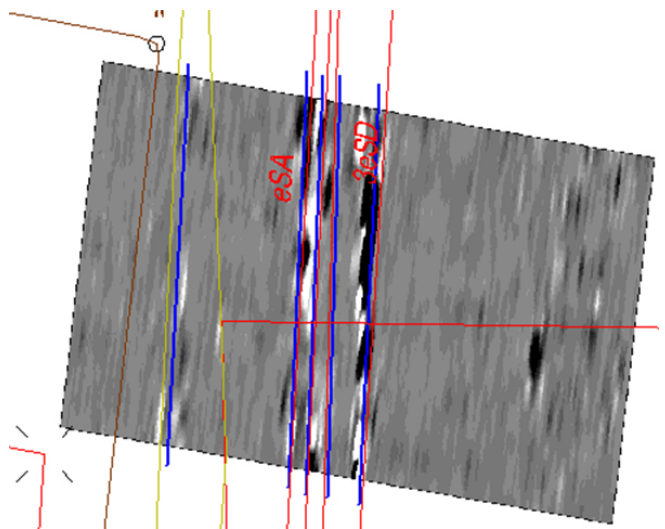

(a) Vectorisation in the local system

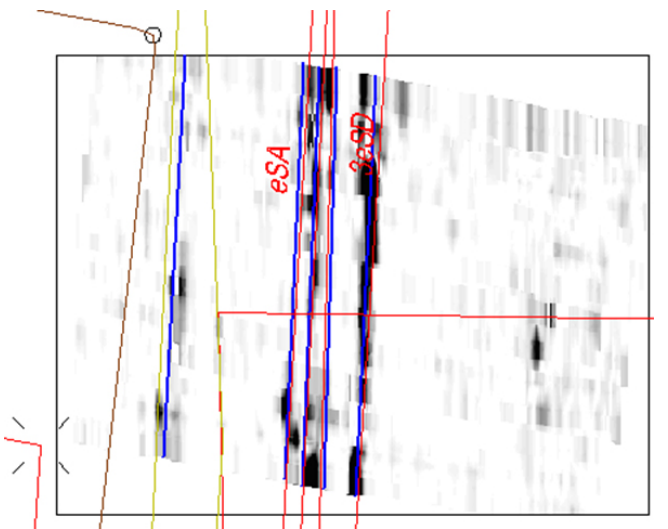

(b) Vectorisation taking account of georeference of the echograms

Figure 15. Vectorisation of the utility line axes based on the slice generated from the 3D model

adjustments.

Both the experiment carried out in Mydlniki and the attempt to verify the compliance of the profile of the utility network captured from cartographic materials, confirmed the legitimacy of the requirement of the PAS128 specification to supplement GPR surveys by using a different indirect method. The geotechnical conditions of the studied medium limited the possibility of capturing complete data. Despite the high resolution of the surveys and the use of appropriate filtration algorithms, it turned out to be impossible to clearly identify all the net- works marked on the map. An additional utility line, not exhibited in the GESUT database, was identified, which would not have been possible using only EM detectors. A new profile of the axis for the gnD300 gas pipe was determined, shifted from the original one by approximately $0.5 \mathrm{~m}$ towards the east. This is probably related to the gross errors, which the data captured based on the digitalisation of the archival materials are encumbered with. It is a similar case with the koD300 sewage pipe appearing in the GESUT database. It was not identified in the echograms despite the large diameter of the pipe, which usually 
produces a clearly visible signal response. In this case, there is only a possibility of pointing out an incorrect profile of the pipeline specified in the documentation, without indicating its correct location.

The structure of the land at Reymonta street had a negative effect on the results of GPR surveys, also for the DS2000 system. However, the importance of using two frequencies of the emitted EM wave should be noted. The occurrence of strong reflections in the surface layer significantly weakened the signal of the $700 \mathrm{MHz}$ antenna already in the initial stage of penetration. However, the information about the depths below $1 \mathrm{~m}$ was supplemented by the echograms of the $250 \mathrm{MHz}$ antenna.

A different look at the results of the surveys and processing of the GPR data may be the key to developing new methods of capturing information on the profiles of underground utility networks. In the future, they may considerably improve the process of identifying the subsoil for infrastructural or construction investment or the verification of the Polish GESUT database. The manufacturers of the GPR equipment should turn their attention to the need for localisation in global systems, which would open a new direction of activities for surveyors. However, it is important to be aware of how the detection methods are operating, based to a large extent on the existing documentation, prerequisites in the form of ground-based facilities and the experience of a person performing surveys and data analysis.

\section{References}

Act (1989). The Act of 17 May 1989 Geodetic and Cartographic Law. Official Journal 1989, No. 30, item 163.

Act (2010). The Act of 4 March 2010 on the Infrastructure for Spatial Information. Official Journal 2010, No. 76, item 489.

BSI (2014). PAS 128: Specification for underground utility detection, verification and location.

Jaw, S. W., Van Son, R., Soon, V. K. H., Schrotter, G., Kiah, R. L. W., Ni, S. T. S., and Yan, J. (2018). The need for a reliable map of utility networks for planning underground spaces. In 17th International Conference on Ground Penetrating Radar (GPR), pages 1-6. IEEE, doi:10.1109/ICGPR.2018.8441625.

Karczewski, J., Ortyl, Ł., and Pasternak, M. (2011). Zarys metody georadarowej. Wydawnictwa AGH.

Lu, Q., Liu, C., Wang, Y., Liu, S., Zeng, Z., Feng, X., and
She, S. (2018). Ground penetrating radar applications in mapping underground utilities. In 17th International Conference on Ground Penetrating Radar (GPR), pages 1-4. IEEE, doi:10.1109/ICGPR.2018.8441659.

Ortyl, Ł. (2006). Badanie przydatności metody georadarowej $w$ geodezyjnej inwentaryzacji struktur i obiektów podpowierzchniowych. PhD thesis, Wydział Geodezji Górniczej i Inżynierii Środowiska AGH, Kraków.

Ortyl, Ł., Kuras, P., Kędzierski, M., and Podstolak, P. (2015). Methods of measurement natural frequency and logarithmic decrement of damping steel chimneys in situations required by Eurocodes (Metody pomiaru częstotliwości drgań własnych i logarytmicznego dekrementu tłumienia kominów stalowych w sytuacjach wymaganych przez Eurokody). Materiaty Budowlane, (9):107-109, doi:10.15199/33.2015.09.42.

Ortyl, Ł. and Owerko, T. (2007). The correction of areas drop and frequency of determining a position by the global positioning system or TCRA total stations in the process of spatial localisation of the objects detected by a georadar (Korekta wpływu deniwelacji terenu oraz częstotliwości wyznaczenia pozycji przez system GNSS lub tachymetry TCA w procesie przestrzennej lokalizacji obiektów wykrywanych georadarem). Zeszyty Naukowe. Górnictwo/Politechnika Śląska, (278):335-352.

Radzevicius, S. J. and Daniels, J. J. (2000). Ground penetrating radar polarization and scattering from cylinders. Journal of Applied Geophysics, 45(2):111-125, doi:10.1016/S09269851(00)00023-9.

Regulation (2011). Regulation of the Minister of Internal Affairs and Administration of 9 November 2011 on technical standards for performing geodetic measurements and for the elaborate and transfer of the results of these measurements to the national geodetic and cartographic resources. Official Journal 2011, No. 263, item 1572.

Regulation (2015). Regulation of the Minister of Administration and Digitalization of 21 October 2015 on the poviat and national GESUT databases. Official Journal 2015, item 1983.

Reichel, B. (2017). Assessment of the use of groundpenetrating radar to detect underground installation systems in winter conditions (Ocena zastosowania georadaru do wykrywania podziemnych instalacji budowlanych w warunkach zimowych). Przegląd Geologiczny, 65(10/2):790795 . 